\title{
Pacific
}

Journal of

Mathematics

\section{PERIODS AND LEFSCHETZ ZETA FUNCTIONS}

\author{
Josefina CASASAyas, Jaume Llibre And ANa NunEs
}

Volume 165 No. 1 


\title{
PERIODS AND LEFSCHETZ ZETA FUNCTIONS
}

\section{Josefina Casasayas, JAume Llibre and ANA Nunes}

\begin{abstract}
The goal of this paper is to obtain information on the set of periods for a transversal self-map of a compact manifold from the associated Lefschetz zeta function in the case when all its zeros and poles are roots of unity.
\end{abstract}

1. Introduction and statement of the results. One of the most useful theorems for proving the existence of fixed points or, more generally, periodic points of a transversal self-map $f$ of a compact manifold is the Lefschetz fixed point theorem. When studying the periodic points of $f$, i.e., the set

$\operatorname{Per}(f)=\{m \in \mathbb{N}: f$ has a periodic orbit of minimal period $m\}$,

it is convenient to use the Lefschetz zeta function of $f, Z_{f}(t)$, which is a generating function for the Lefschetz numbers of all iterates of $f$. The function $Z_{f}(t)$ is rational in $t$ and can be computed from the homological invariants of $f$ (see $\S 3$ ).

We shall study $C^{1}$ self-maps $f$ of a compact manifold which have only transversal periodic points, so called because the graph of $\mathrm{fm}^{\mathrm{m}}$ is transverse to the diagonal for all $m>0$. The main contribution of this paper is the study of the periodic orbits of $f$ when its Lefschetz zeta function has a finite factorization into terms of the form $\left(1 \pm t^{n}\right)^{ \pm 1}$. A key point is the introduction of the notion of irreducible factor (see $\S 3$ for a precise definition). Our main result is the following.

TheOREM A. Let $f: M \rightarrow M$ be a transversal map of a compact manifold. Suppose that all the zeros and poles of its Lefschetz zeta function $Z_{f}(t)$ are roots of unity, and that $Z_{f}(t)$ has an irreducible factor of the form $\left(1 \pm t^{n}\right)^{ \pm 1}$.

(a) If $n$ is odd then $n \in \operatorname{Per}(f)$.

(b) If $n$ is even then $\left\{\frac{n}{2}, n\right\} \cap \operatorname{Per}(f) \neq \varnothing$.

The proof of this theorem will be given in $\S 3$. From Theorem A it follows that each irreducible factor of the form $\left(1 \pm t^{n}\right)^{ \pm 1}$ of the 
Lefschetz zeta function forces at least one period ( $n$ if $n$ is odd, $n / 2$ or $n$ if $n$ is even).

The set of periods obtained in this way will be called the forced set of periods of $f$ and will be denoted by $\operatorname{FSP}(f)$.

As an application of Theorem $A$ and the algebraic results derived in $\S 2$, we obtain an upper bound for the cardinal and for the maximum period of the forced set of periods (see Corollaries 3.3 and 3.4).

Our main basic assumption throughout this work is that all the zeros and poles of the Lefschetz zeta function associated to $f: M \rightarrow M$ are roots of unity (for different results under similar assumptions see Franks [F1], [F3], Fried [Fr], Matsuoka [Mt] and [CLN]). There are three interesting classes of transversal maps which satisfy our basic assumption. First, the set of maps whose set of periods $\operatorname{Per}(f)$ is finite (see Theorem 6 of [Fr]). Second, the self-maps of compact connected surfaces with $\operatorname{Per}(f)$ finite or $h(f)=0$, see Corollaries 4.3 and 4.4. Finally, the self-maps of the $n$-dimensional torus with $\operatorname{Per}(f)$ finite or $h(f)=0$, see Corollaries 5.1 and 5.2.

2. Cyclotomic polynomials. As usual, we shall use the notation $c_{n}(t)$ for the $n$th cyclotomic polynomial given by

$$
c_{n}(t)=\frac{1-t^{n}}{\prod_{d \mid n, d<n} c_{d}(t)}
$$

for $n \in \mathbb{N} \backslash\{1\}$ and $c_{1}(t)=1-t$.

Notice that all the zeros of $c_{n}(t)$ are roots of unity.

A proof of the next proposition may be found in [L].

Proposition 2.1. Let $\xi$ be a primitive $n$th root of unity and $P(t)$ a polynomial with rational coefficients. If $P(\xi)=0$ then $c_{n}(t) \mid P(t)$.

Clearly, the degree $\varphi(n)$ of $c_{n}(t)$ verifies

$$
n=\sum_{d \mid n} \varphi(d)
$$

and so $\varphi(n)$ is the Euler function, which may be computed through

$$
\varphi(n)=n \prod_{\substack{p \mid n \\ p \text { prime }}}\left(1-\frac{1}{p}\right) .
$$

Hence, if $n=p_{1}^{\alpha_{1}} \cdots p_{k}^{\alpha_{k}}$ is the prime decomposition of $n$, then

$$
\varphi(n)=\prod_{i=1}^{k} p_{i}^{\alpha_{i}-1}\left(p_{i}-1\right)
$$


TABLE 1

\begin{tabular}{llll}
\hline$c_{1}(t)=1-t$ & $c_{2}(t)=1+t$ & $c_{3}(t)=\frac{1-t^{3}}{1-t}$ \\
$c_{4}(t)=1+t^{2}$ & $c_{5}(t)=\frac{1-t^{5}}{1-t}$ & $c_{6}(t)=\frac{1+t^{3}}{1+t}$ \\
$c_{7}(t)=\frac{1-t^{7}}{1-t}$ & $c_{8}(t)=1+t^{4}$ & $c_{9}(t)=\frac{1-t^{9}}{1-t^{3}}$ \\
$c_{10}(t)=\frac{1+t^{5}}{1+t}$ & $c_{11}(t)=\frac{1-t^{11}}{1-t}$ & $c_{12}(t)=\frac{1+t^{6}}{1+t^{2}}$ \\
$c_{13}(t)=\frac{1-t^{13}}{1-t}$ & $c_{14}(t)=\frac{1+t^{7}}{1+t}$ & $c_{15}(t)=\frac{\left(1-t^{15}\right)(1-t)}{\left(1-t^{3}\right)\left(1-t^{5}\right)}$ \\
$c_{16}(t)=1+t^{8}$ & $c_{17}(t)=\frac{1-t^{17}}{1-t}$ & $c_{18}(t)=\frac{1+t^{9}}{1+t^{3}}$ \\
$c_{19}(t)=\frac{1-t^{19}}{1-t}$ & $c_{20}(t)=\frac{1+t^{10}}{1+t^{2}}$ & $c_{21}(t)=\frac{\left(1-t^{21}\right)(1-t)}{\left(1-t^{3}\right)\left(1-t^{7}\right)}$ \\
$c_{22}(t)=\frac{1+t^{11}}{1+t}$ & $c_{23}(t)=\frac{1-t^{23}}{1-t}$ & $c_{24}(t)=\frac{1+t^{12}}{1+t^{4}}$ \\
$c_{25}(t)=\frac{1-t^{25}}{1-t^{5}}$ & $c_{26}(t)=\frac{1+t^{13}}{1+t}$ & $c_{27}(t)=\frac{1-t^{27}}{1-t^{9}}$ \\
$c_{28}(t)=\frac{1+t^{14}}{1+t^{2}}$ & $c_{29}(t)=\frac{1-t^{29}}{1-t}$ & $c_{30}(t)=\frac{\left(1+t^{15}\right)(1+t)}{\left(1+t^{3}\right)\left(1+t^{5}\right)}$ \\
\hline
\end{tabular}

In Table 1 we present a list of the first 30 cyclotomic polynomials and their degrees. The following rules follow easily from the definition of cyclotomic polynomials and their properties (see [L]).

$$
\begin{aligned}
& p \text { prime } \Rightarrow c_{p}(t)=\frac{1-t^{p}}{1-t}, \\
& p=2^{n} \Rightarrow c_{p}(t)=1+t^{2^{n-1}} \text {, } \\
& p=2 r, r \text { odd } \Rightarrow c_{p}(t)=c_{r}(-t), \\
& p=2^{n} r \text {, rodd, } n>1 \Rightarrow c_{p}(t)=c_{2 r}\left(t^{2^{n-1}}\right) \text {, } \\
& p=p_{1} p_{2}, p_{1}, p_{2} \text { prime } \Rightarrow c_{p}(t)=\frac{c_{p_{1}}\left(t^{p_{2}}\right)}{c_{p_{1}}(t)}=\frac{c_{p_{2}}\left(t^{p_{1}}\right)}{c_{p_{2}}(t)}, \\
& p=p_{1}^{\alpha}, p_{1} \text { prime } \Rightarrow c_{p}(t)=c_{p_{1}}\left(t^{p_{1}^{\alpha-1}}\right)=\frac{1-t^{p_{1}^{\alpha}}}{1-t_{1}^{p_{1}^{\alpha-1}}}, \\
& c_{p_{1}^{\alpha_{1}} \cdots p_{k}^{\alpha_{k}}}(t)=c_{p_{1} \cdots p_{k}}\left(t^{p_{1}^{\alpha_{1}-1} \cdots p_{k}^{\alpha_{k}-1}}\right),
\end{aligned}
$$




$$
p \text { prime }, p \nmid r \Rightarrow c_{p r}(t)=\frac{c_{r}\left(t^{p}\right)}{c_{r}(t)} \text {. }
$$

LEMMA 2.2. Let $n=p_{1}^{\alpha_{1}} \cdots p_{k}^{\alpha_{k}}$ be the prime decomposition of $n \in$ $\mathbb{N}$. Then

(2.10)

$$
\begin{aligned}
c_{p_{1}^{\alpha_{1}} \ldots p_{k}^{\alpha_{k}}}(t)= & c_{p_{k}}\left(t^{p_{k}^{\alpha_{k}-1}}\right)^{(-1)^{k-1}} \\
& \cdot \prod_{j=1}^{k-1} \prod_{1 \leq i_{1}<\cdots<i_{j} \leq k-1} c_{p_{k}}\left(\left(t^{p_{i_{1}} \ldots p_{i_{j}}}\right)^{p_{1}^{\alpha_{1}-1} \cdots p_{k}^{\alpha_{k}-1}}\right)^{(-1)^{k-1-j}} .
\end{aligned}
$$

Proof. Using property (2.8), it is enough to show that

$$
\begin{aligned}
c_{p_{1} \ldots p_{k}}(t)= & c_{p_{k}}(t)^{(-1)^{k-1}} \\
& \cdot \prod_{j=1}^{k-1} \prod_{1 \leq i_{1}<\cdots<i_{j} \leq k-1} c_{p_{k}}\left(t^{p_{i_{1}} \cdots p_{i_{j}}}\right)^{(-1)^{k-1-j}},
\end{aligned}
$$

and we shall prove $(2.11)$ by induction with respect to $k \in \mathbb{N}$. For $k=1$ it holds trivially. Suppose $k=2$. By property (2.6) we have

$$
c_{p_{1} p_{2}}(t)=c_{p_{2}}(t)^{-1} c_{p_{2}}\left(t^{p_{1}}\right),
$$

and so (2.11) holds for $k=2$. Suppose now that (2.11) holds for some $k \in \mathbb{N}, k \geq 2$, and consider $c_{p_{1} \ldots p_{k+1}}(t)$ with $p_{1}<\cdots<p_{k+1}$. Then, applying successively (2.9) and the induction hypothesis,

$$
\begin{aligned}
c_{p_{1} \ldots p_{k+1}}(t)= & \frac{c_{p_{2} \ldots p_{k+1}}\left(t^{p_{1}}\right)}{c_{p_{2} \ldots p_{k+1}}(t)} \\
= & \frac{c_{p_{k+1}}\left(t^{p_{1}}\right)^{(-1)^{k-1}}}{c_{p_{k+1}}(t)^{(-1)^{k-1}}} \\
& \cdot \prod_{j=1}^{k-1} \prod_{2 \leq i_{1}<\cdots<i_{j} \leq k}\left[\frac{c_{p_{k+1}}\left(t^{p_{1} p_{i_{1}} \ldots p_{i_{j}}}\right)}{c_{p_{k+1}}\left(t^{p_{i_{1}} \ldots p_{i_{j}}}\right)}\right]^{(-1)^{k-1-j}} \\
= & c_{p_{k+1}}(t)^{(-1)^{k}} c_{p_{k+1}}\left(t^{p_{1}}\right)^{(-1)^{k-1}} \\
& \cdot \prod_{j=1}^{k-1} \prod_{2 \leq i_{1}<\cdots<i_{j} \leq k} c_{p_{k+1}}\left(t^{p_{1} p_{i_{1}} \ldots p_{i_{j}}}\right)^{(-1)^{k-j-1}}
\end{aligned}
$$

$$
\cdot c_{p_{k+1}}\left(t^{p_{i_{1}} \ldots p_{i_{j}}}\right)^{(-1)^{k-j}}
$$


Now it is easy to check that (2.12) is equal to

$$
c_{p_{k+1}}(t)^{(-1)^{k}} \prod_{j=1}^{k-1} \prod_{1 \leq i_{1}<\cdots<i_{j} \leq k} c_{p_{k+1}}\left(t^{p_{i_{1}} \ldots p_{i_{j}}}\right)^{(-1)^{k-j}} .
$$

Hence, (2.11) holds for $k+1$ and the lemma is proved.

Proposition 2.3. Let $c_{n}(t)$ be of degree $\varphi(n)>2$. Then $c_{n}(t)$ can be written as

$$
c_{n}(t)=\prod_{i=1}^{m}\left(1-\sigma_{1}(i) t^{q_{i}}\right)^{\sigma_{2}(i)},
$$

where $m \leq \varphi(n) / 2, q_{i} \in \mathbb{N}$ and $\sigma_{1}(i), \sigma_{2}(i) \in\{-1,1\}$ for $i=$ $1, \ldots, m$. Moreover, $q=\max _{i} q_{i}$ is smaller than or equal to $n / 2$ if $n$ is even or $n$ if $n$ is odd.

Proof. Let $n=p_{1}^{\alpha_{1}} \cdots p_{k}^{\alpha_{k}}$ be the prime decomposition of $n$. The case $k=1$ is obvious from (2.7) and (2.3). Suppose first that $k \geq 2$ and that none of the $p_{i}$ is 2 . From Lemma 2.2 and (2.2), $c_{n}(t)$ factorizes in the form (2.13) with

$$
m=2 \sum_{j=0}^{k-1}\left(\begin{array}{c}
k-1 \\
j
\end{array}\right)=2^{k}
$$

From (2.1)

$$
\varphi(n)=\prod_{i=1}^{k} p_{i}^{\alpha_{i}-1}\left(p_{i}-1\right) \geq 2 \cdot 4 \cdot 2^{k-2}=2^{k+1},
$$

and the result follows with $\sigma_{1}(i)=+1$ for $i=1, \ldots, m$.

Suppose now that $k \geq 2$ and that one of the $p_{i}$ is 2. Applying Lemma 2.2 with $p_{k}=2$ and (2.3), $c_{n}(t)$ factorizes in the form (2.13) with $\sigma_{1}(i)=-1$ for $i=1, \ldots, m$ and

$$
m=\sum_{j=0}^{k-1}\left(\begin{array}{c}
k-1 \\
j
\end{array}\right)=2^{k-1} .
$$

But for $k \geq 3$

$$
\varphi(n)=\prod_{i=1}^{k} p_{i}^{\alpha_{i}-1}\left(p_{i}-1\right) \geq 2 \cdot 8 \cdot 2^{k-3}=2^{k+1}
$$


TABLE 2

\begin{tabular}{rrrrrrrrrr}
\hline$d$ & $n_{0}(d)$ & $d$ & $n_{0}(d)$ & $d$ & $n_{0}(d)$ & $d$ & $n_{0}(d)$ & $d$ & $n_{0}(d)$ \\
\hline 1 & 2 & 2 & 6 & 3 & 9 & 4 & 12 & 5 & 15 \\
6 & 18 & 7 & 21 & 8 & 30 & 9 & 33 & 10 & 37 \\
11 & 41 & 12 & 45 & 13 & 48 & 14 & 52 & 15 & 56 \\
16 & 60 & 17 & 63 & 18 & 67 & 19 & 71 & 20 & 75 \\
21 & 78 & 22 & 82 & 23 & 86 & 24 & 90 & 25 & 93 \\
26 & 97 & 27 & 101 & 28 & 105 & 29 & 108 & 30 & 112 \\
31 & 116 & 32 & 120 & 33 & 123 & 34 & 127 & 35 & 131 \\
36 & 135 & 37 & 138 & 38 & 142 & 39 & 146 & 40 & 150 \\
41 & 153 & 42 & 157 & 43 & 161 & 44 & 165 & 45 & 168 \\
46 & 172 & 47 & 176 & 48 & 210 & 49 & 214 & 50 & 218 \\
51 & 223 & 52 & 227 & 53 & 231 & 54 & 236 & 55 & 240 \\
56 & 245 & 57 & 249 & 58 & 253 & 59 & 258 & 60 & 262 \\
61 & 266 & 62 & 271 & 63 & 275 & 64 & 280 & 65 & 284 \\
66 & 288 & 67 & 293 & 68 & 297 & 69 & 301 & 70 & 306 \\
71 & 310 & 72 & 315 & 73 & 319 & 74 & 323 & 75 & 328 \\
76 & 332 & 77 & 336 & 78 & 341 & 79 & 345 & 80 & 350 \\
81 & 354 & 82 & 358 & 83 & 363 & 84 & 367 & 85 & 371 \\
86 & 376 & 87 & 380 & 88 & 385 & 89 & 389 & 90 & 393 \\
91 & 398 & 92 & 402 & 93 & 406 & 94 & 411 & 95 & 415 \\
96 & 420 & 97 & 424 & 98 & 428 & 99 & 433 & 100 & 437 \\
\hline
\end{tabular}

and the result follows as before. If $k=2, m=2$. But $\varphi(n)$ is even and hence by hypothesis $\varphi(n) \geq 4$. So, again, $m \leq \varphi(n) / 2$.

From the definition of $c_{n}(t)$ it is clear that $q \leq n$, and from (2.4) and (2.5) it follows that $q \leq n / 2$ if $n$ is even.

Given $d \in \mathbb{N}$, let $n_{0}(d)$ be defined by

$$
n_{0}(d)=\left[d \prod_{i=1}^{k} \frac{p_{i}}{p_{i}-1}\right]
$$

where [.] denotes the integer part function and $k \in \mathbb{N}$ is the greatest number of consecutive primes $p_{i}$ such that $p_{1}=2$ and $\left(p_{1}-1\right) \cdots\left(p_{k}-1\right) \leq d$. See Table 2 for the first one hundred values of $n_{0}(d)$.

Proposition 2.4. Given $d \in \mathbb{N}$, there exists $n_{0} \in \mathbb{N}$ such that for $n>n_{0}, \varphi(n)>d$. Moreover, $n_{0}(d)$ is the best possible lower bound for $n_{0}$. 
Proof. For $d=8$ we obtain $n_{0}(d)=30$ and $\varphi(30)=8$. So, (2.14) gives the best possible lower bound.

Let $d \in \mathbb{N}$ and $n_{0} \in \mathbb{N}$ be given by (2.14). Let $n$ be greater than $n_{0}$ so that

$$
n>\left[d \prod_{i=1}^{k} \frac{p_{i}}{p_{i}-1}\right] \geq \prod_{i=1}^{k} p_{i}
$$

and let $n=p_{1}^{\alpha_{1}} \cdots p_{j}^{\alpha_{J}}$ be the prime decomposition of $n$. In order to prove that $\varphi(n)>d$ we shall consider two cases. Suppose first that $j>k$. Then, from $(2.1)$

$$
\varphi(n)=\prod_{i=1}^{j} p_{n_{i}}^{\alpha-1}\left(p_{n_{i}}-1\right) \geq \prod_{i=1}^{j}\left(p_{n_{t}}-1\right) \geq \prod_{i=1}^{k+1}\left(p_{i}-1\right)>d .
$$

Suppose now that $j \leq k$. Then

$$
\begin{aligned}
\varphi(n)=\prod_{i=1}^{j} p_{n_{i}}^{\alpha_{i}-1}\left(p_{n_{i}}-1\right) & \geq n \prod_{i=1}^{j} \frac{p_{n_{i}}-1}{p_{n_{i}}} \\
& >d \prod_{i=1}^{k} \frac{p_{i}}{p_{i}-1} \prod_{i=1}^{j} \frac{p_{n_{t}}-1}{p_{n_{t}}} \geq d,
\end{aligned}
$$

because the $p_{i}$ are consecutive primes and so, for $i=1, \ldots, j$

$$
\frac{p_{i}}{p_{i}-1} \cdot \frac{p_{n_{i}}-1}{p_{n_{t}}} \geq 1
$$

3. Periods forced by the Lefschetz zeta function. Let $M$ be a compact manifold. A map $f: M \rightarrow M$ is called transversal if

(1) $f$ is of class $C^{1}$,

(2) $f(M) \subset \operatorname{Int}(M)$,

(3) for every periodic orbit $\gamma$ of period $p(\gamma), x \in \gamma$ and $m \in \mathbb{N}$

$$
\operatorname{det}\left(D f^{m p(\gamma)}(x)-I\right) \neq 0 .
$$

Let $f: M \rightarrow M$ be a transversal map. Denote by PO the set of periodic orbits of $f$ and, given $\gamma \in \mathrm{PO}$, by $p(\gamma)$ the minimal period of $\gamma$. Following the notation introduced in [CLN] we define $u_{+}(\gamma)$ (resp. $u_{-}(\gamma)$ ) as the number of real eigenvalues of $D f^{p(\gamma)}(x), x \in \gamma$, which are strictly greater than one (resp. strictly smaller than one). We also define the following subsets of PO: 


$$
\begin{aligned}
& \mathrm{EE}=\left\{\gamma \in \mathrm{PO}: u_{+}(\gamma) \text { and } u_{-}(\gamma) \text { are even }\right\}, \\
& \mathrm{EO}=\left\{\gamma \in \mathrm{PO}: u_{+}(\gamma) \text { is even and } u_{-}(\gamma) \text { is odd }\right\}, \\
& \mathrm{OE}=\left\{\gamma \in \mathrm{PO}: u_{+}(\gamma) \text { is odd and } u_{-}(\gamma) \text { is even }\right\}, \\
& \mathrm{OO}=\left\{\gamma \in \mathrm{PO}: u_{+}(\gamma) \text { and } u_{-}(\gamma) \text { are odd }\right\} .
\end{aligned}
$$

Also, for each odd $r \geq 1$ and $n \geq 0$ let $\mathrm{PO}\left(2^{n} r\right)$ be the set

$$
\mathrm{PO}\left(2^{n} r\right)=\left\{\gamma \in \mathrm{PO}: p(\gamma)=2^{n} r\right\},
$$

and denote by $\mathrm{EE}_{2^{n} r}$ (resp. $\mathrm{EO}_{2^{n} r}, \mathrm{OE}_{2^{n} r}, \mathrm{OO}_{2^{n} r}$ ) the cardinal of $\mathrm{EE} \cap \mathrm{PO}\left(2^{n} r\right)$ (resp. $\left.\mathrm{EO} \cap \mathrm{PO}\left(2^{n} r\right), \mathrm{OE} \cap \mathrm{PO}\left(2^{n} r\right), \mathrm{OO} \cap \mathrm{PO}\left(2^{n} r\right)\right)$.

Denote by $\mu: \mathbb{N} \rightarrow\{-1,0,1\}$ the Möbius function defined by $\mu(1)=1$ and by the following rule: if $n=p_{1}^{k_{1}} \cdots p_{j}^{k_{j}}$ is the prime decomposition of $n, \mu(n)=0$ if $k_{i}>1$ for some $i \in\{1, \ldots, j\}$, and $\mu(n)=(-1)^{j}$ otherwise. Denote by $\left\{\alpha_{n}\right\}_{n \in \mathbb{N}}$ the sequence of nonnegative integers defined by

$$
\alpha_{n}=\frac{1}{n} \sum_{k \mid n} \mu\left(\frac{n}{k}\right),
$$

and, for each positive odd $r$ and each nonnegative $m$, let

$$
\beta_{2^{m} r}=\sum_{k=0}^{m} \alpha_{2^{k} r}
$$

Given a continuous self-map of a compact manifold $M$ of dimension $n$, its Lefschetz number is defined as

$$
L(f)=\sum_{k=0}^{n}(-1)^{k} \operatorname{tr}\left(f_{* k}\right),
$$

where $f_{* k}: H_{k}(M ; \mathbb{Q}) \rightarrow H_{k}(M ; \mathbb{Q})$ is the endomorphism induced by $f$ on the $k$ th rational homology group of $M$. The Lefschetz fixed point theorem says that if $L(f) \neq 0$ then $f$ has a fixed point. For the purpose of studying the set $\operatorname{Per}(f)$, it is useful to consider the Lebschetz zeta function

$$
Z_{f}(t)=\exp \left(\sum_{m=1}^{\infty} \frac{L\left(f^{m}\right)}{m} t^{m}\right),
$$

which is a generating function for the Lefschetz numbers of all iterates of $f$ and can be computed from the homological endomorphisms $f_{* k}$ 
of $f$ as follows:

$$
Z_{f}(t)=\prod_{k=0}^{n} \operatorname{det}\left(I_{j_{k}}-t f_{* k}\right)^{(-1)^{k+1}}
$$

where $j_{k}=\operatorname{dim}_{\mathbb{Q}} H_{k}(M ; \mathbb{Q})$, see [F2].

If $f: M \rightarrow M$ is transversal, then the Lefschetz numbers of the iterates of $f$ are related in a simple way to the periodic points of $f$, see [F1] for more details.

With this notation, we have the following theorem, which will be used in subsequent proofs.

TheOREM 3.1 ([CLN]). Let $f: M \rightarrow M$ be a transversal map such that its Lefschetz zeta function is of the form

$$
Z_{f}(t)=\prod_{p \in P} \prod_{i=1}^{N_{p}}\left(1-\sigma_{1}(i, p) t^{p}\right)^{\sigma_{2}(i, p)},
$$

where $P$ is a finite subset of $\mathbb{N}$, the $N_{p}$ are natural numbers and $\sigma_{1}(i, p), \sigma_{2}(i, p) \in\{-1,1\}$. Then, for each odd integer $r \geq 1$ and $m=0,1,2, \ldots$ we have

$$
\sum_{k=0}^{m} \mathrm{EE}_{2^{k} r}+\mathrm{EO}_{2^{m} r}+\gamma(r, m)=\mathrm{OO}_{2^{m} r}+\sum_{k=0}^{m} \mathrm{OE}_{2^{k} r},
$$

where

(3.6)

$$
\begin{aligned}
\gamma(r, m)= & \sum_{\substack{q \mid r \\
2^{m} q \in P}} \sum_{i=1}^{N_{2^{m} q}} \sigma_{2}\left(i, 2^{m} q\right) \sigma_{1}\left(i, 2^{m} q\right) \beta_{r / q} \\
& +\sum_{j=0}^{m-1} \sum_{\substack{q \mid r \\
2^{j} q \in P}} \sum_{i=1}^{N_{2^{j}}} \sigma_{2}\left(i, 2^{j} q\right)\left(\beta_{2^{m-j} r / q}+\frac{\sigma_{2}\left(i, 2^{j} q\right)-1}{2} \beta_{r / q}\right),
\end{aligned}
$$

and we take the second summand equal zero when $m=0$.

Consider a product of the form

$$
\prod_{p \in P} \prod_{i=1}^{N_{p}}\left(1-\sigma_{1}(i, p) t^{p}\right)^{\sigma_{2}(i, p)},
$$


where $P$ is a finite subset of $\mathbb{N}, N_{p}$ is a natural number for each $p \in P$, and $\sigma_{1}(i, p), \sigma_{2}(i, p) \in\{-1,1\}$. A factor in (3.7) of the form $\left(1-\sigma_{1}(i, p) t^{p}\right)^{\sigma_{2}(i, p)}$ will be called irreducible if it remains after performing the following reductions:

$$
\begin{gathered}
\left(1+t^{p}\right)\left(1+t^{p}\right)^{-1}=1 \\
\left(1-t^{p}\right)\left(1-t^{p}\right)^{-1}=1 \\
\left(1+t^{p}\right)\left(1-t^{p}\right)=1-t^{2 p} \\
\left(1+t^{p}\right)^{-1}\left(1-t^{p}\right)^{-1}=\left(1-t^{2 p}\right)^{-1} \\
\left(1+t^{p}\right)^{-1}\left(1-t^{2 p}\right)=1-t^{p} \\
\left(1+t^{p}\right)\left(1-t^{2 p}\right)^{-1}=\left(1-t^{p}\right)^{-1} \\
\left(1+t^{p}\right)\left(1+t^{2 p}\right)=\left(1-t^{p}\right)^{-1}\left(1-t^{4 p}\right) \\
\left(1+t^{p}\right)^{-1}\left(1+t^{2 p}\right)^{-1}=\left(1-t^{p}\right)\left(1-t^{4 p}\right)^{-1}
\end{gathered}
$$

We remark that the reductions (R1) to (R8) should be performed in correlative order and consequently the whole process of reduction is finite.

THEOREM 3.2. Let $f: M \rightarrow M$ be a transversal map of a compact manifold. Suppose that all the zeros and poles of its Lefschetz zeta function $Z_{f}(t)$ are roots of unity, and that $Z_{f}(t)$ has an irreducible factor of the form $\left(1 \pm t^{n}\right)^{ \pm 1}$.

(a) If $n$ is odd then $n \in \operatorname{Per}(f)$.

(b) If $n$ is even then $\left\{\frac{n}{2}, n\right\} \cap \operatorname{Per}(f) \neq \varnothing$.

Proof. From (3.3) and Proposition 2.1, the Lefschetz zeta function of $f$ is of the form

$$
Z_{f}(t)=\frac{\prod_{i=1}^{N} c_{n_{i}}(t)}{\prod_{i=1}^{Q} c_{q_{i}}(t)} .
$$

Moreover, applying Proposition 2.3 to each $c_{n_{i}}(t), c_{q_{i}}(t), f$ is in the hypothesis of Theorem 3.1 and so, given $r$ odd, equation (3.5) holds for $m=0$. Now, if we have an irreducible factor of the form $\left(1-\sigma_{1} t^{r}\right)^{\sigma_{2}}$, then all the other possible irreducible factors associated to the same power of $t$ are $\left(1-\sigma_{1} t^{r}\right)^{\sigma_{2}}$ or $\left(1+\sigma_{1} t^{r}\right)^{-\sigma_{2}}$ (see reduction rules (R1) to (R4)). Hence,

$$
\left|\sum_{i=1}^{N_{r}} \sigma_{2}(i, r) \sigma_{1}(i, r)\right| \neq 0
$$


because all the terms in the above sum are equal. So, $r \in \operatorname{Per}(f)$ and statement (a) is proved.

Now we shall prove statement (b). From the definition (3.6) of $\gamma(r, m)$ for $m=1,2, \ldots$ we have

$$
\begin{aligned}
\gamma(r, m)-\gamma(r, m-1)= & \sum_{i=1}^{N_{2^{m}}} \sigma_{2}\left(i, 2^{m} r\right) \sigma_{1}\left(i, 2^{m} r\right) \\
& +\sum_{i=1}^{N_{2^{m-1} r}} \sigma_{2}\left(i, 2^{m-1} r\right)\left(\frac{1-\sigma_{1}\left(i, 2^{m-1} r\right)}{2}\right),
\end{aligned}
$$

and subtracting (3.5) for $m$ and $m-1$ we obtain

$$
\begin{aligned}
& \mathrm{EE}_{2^{m} r}+\mathrm{EO}_{2^{m} r}-\mathrm{EO}_{2^{m-1} r}+\gamma(r, m)-\gamma(r, m-1) \\
& \quad=\mathrm{OE}_{2^{m} r}+\mathrm{OO}_{2^{m} r}-\mathrm{OO}_{2^{m-1} r} .
\end{aligned}
$$

Suppose $n=2^{m} r, m \geq 1, r$ odd. Consider an irreducible factor of the form $1-t^{2^{m} r}$. The proof for the other possible irreducible factors $\left(1-t^{2^{m} r}\right)^{-1}, 1+t^{2^{m} r}$ and $\left(1+t^{2^{m} r}\right)^{-1}$ is analogous. For this factor we have $\sigma_{1}\left(\cdot, 2^{m} r\right) \sigma_{2}\left(\cdot, 2^{m} r\right)=1$ and so all the factors of the form

$$
\left(1-\sigma_{1}\left(i, 2^{m} r\right) t^{2^{m} r}\right)^{\sigma_{2}\left(i, 2^{m} r\right)}
$$

that persist after reductions of the type (R1)-(R4) verify $\sigma_{1}\left(i, 2^{m} r\right)$ - $\sigma_{2}\left(i, 2^{m} r\right)=1$. Moreover, the factors of the form

$$
\left(1-\sigma_{1}\left(i, 2^{m-1} r\right) t^{2^{m-1}} r\right)^{\sigma_{2}\left(i, 2^{m-1} r\right)}
$$

that can coexist with $1-t^{2^{m} r}$ satisfy

$$
\sigma_{2}\left(i, 2^{m-1} r\right)\left(\frac{1-\sigma_{1}\left(i, 2^{m-1} r\right)}{2}\right) \in\{0,1\}
$$

due to the reduction (R5). Then $|\gamma(r, m)-\gamma(r, m-1)|>0$ and equation (3.8) implies that if $2^{m-1} r \notin \operatorname{Per}(f)$ then $2^{m} r \in \operatorname{Per}(f)$.

We remark that the proof for the irreducible factors $\left(1-t^{2^{m} r}\right)^{-1}$, $1+t^{2^{m} r}$ and $\left(1+t^{2^{m} r}\right)^{-1}$ uses the reductions (R6), (R7) and (R8), respectively, and of course also (R1) to (R4).

Let $P(t) / Q(t)$ be a rational function. We define the order of $P(t) / Q(t)$, denoted by $\operatorname{order}(P(t) / Q(t))$, as the number

$$
\left[\frac{\text { degree } P(t)+\text { degree } Q(t)}{2}\right]+1 \text {. }
$$


COROLlARY 3.3. Let $f: M \rightarrow M$ be a transversal map of a compact manifold. Suppose that all the zeros and poles of $Z_{f}(t)$ are roots of unity. Then

$$
\operatorname{Card}(\operatorname{FSP}(f)) \leq \operatorname{order}\left(Z_{f}(t)\right)
$$

Proof. Since the Lefschetz zeta function of $f, Z_{f}(t)$, is rational, we may write $Z_{f}(t)=P(t) / Q(t)$ with $P(t)$ and $Q(t)$ polynomials.

By Proposition 2.1, $P(t)$ and $Q(t)$ factorize as the product of cyclotomic polynomials. We shall split $P(t)$ (resp. $Q(t)$ ) as a product $P(t)=P_{1}(t) P_{2}(t)$ (resp. $Q(t)=Q_{1}(t) Q_{2}(t)$ ), where $P_{1}(t)$ (resp. $\left.Q_{1}(t)\right)$ factorizes as a product of cyclotomic polynomials of degree strictly greater than 2 and $c_{4}(t)$, and $P_{2}(t)$ (resp. $Q_{2}(t)$ ) contains the remaining factors, i.e. all the $c_{i}(t)$ for $i \in\{1,2,3,6\}$. Since the reduction process given by rules $(\mathrm{R} 1)-(\mathrm{R} 8)$ does not increase the number of factors of the form $\left(1 \pm t^{n}\right)^{ \pm 1}$, from Proposition 2.3 and Theorem 3.2 it follows that the cardinal of the forced set of periods associated to the factors $P_{1}(t)$ and $Q_{1}(t)$ is smaller than or equal to (degree $P_{1}(t)+$ degree $\left.Q_{1}(t)\right) / 2$. Notice that this upper bound is an integer number.

To conclude the proof, it is enough to show that the contribution of the remaining factors $\left(P_{2}(t)\right.$ and $\left.Q_{2}(t)\right)$ to the cardinal of the forced set of periods is smaller than or equal to

$$
C=\left[\frac{\text { degree } P_{2}(t)+\text { degree } Q_{2}(t)}{2}\right]+1 \text {. }
$$

Notice that $P_{2}(t) / Q_{2}(t)$ is a rational function of the form $c_{1}(t)^{a_{1}}$ - $c_{2}(t)^{a_{2}} c_{3}(t)^{a_{3}} c_{6}(t)^{a_{6}}$, where the $a_{i} \in \mathbb{Z}$. The forced set of periods $F$ associated to a product of this form is contained in $\{1,3\}$. To prove that $\operatorname{Card}(F) \leq C$ it is enough to consider the case $\operatorname{Card}(F)=2$ because $C \geq 1$. But if $3 \in F$ then $\left|a_{3}\right|+\left|a_{6}\right| \neq 0$, and hence $C \geq 2$ (see Table 1).

Let $n \in \mathbb{N}$. We denote by $S(n)$ the set $\{1,2, \ldots, n\}$.

Corollary 3.4. Assume that we are in the hypotheses of Corollary 3.3. Let $d$ be the maximum of the degrees of $P(t)$ and $Q(t)$, where $P(t) / Q(t)=Z_{f}(t)$. Then

$\operatorname{FSP}(f) \cap\{n \in \mathbb{N}: n$ is odd $\}$

$$
\subset \begin{cases}S\left(n_{0}(d)\right) & \text { if } n_{0}(d) \text { is odd, } \\ S\left(n_{0}(d)-1\right) & \text { if } n_{0}(d) \text { is even. }\end{cases}
$$


$\operatorname{FSP}(f) \cap\{n \in \mathbb{N}: n$ is even $\}$

$$
\subset \begin{cases}S\left(2 n_{0}(d)\right) & \text { if } n_{0}(d) \text { is odd }, \\ S\left(2 n_{0}(d)-2\right) & \text { if } n_{0}(d) \text { is even. }\end{cases}
$$

Proof. By Proposition 2.1, $P(t)$ and $Q(t)$ factorize as products of cyclotomic polynomials. From Proposition 2.4 it follows that if the cyclotomic polynomial $c_{n}(t)$ appears in the factorization of $P(t)$ or $Q(t)$, then $n \leq n_{0}(d)$. Now, the maximum power of $t$ in the decomposition (2.13) of $c_{n}(t)$ is smaller than or equal to $n / 2$ (resp. $n$ ) if $n$ is even (resp. odd). Consequently, before applying the reduction procedure given by rules (R1) to (R8), the maximum power of $t$ which appears in the factors $\left(1 \pm t^{j}\right)^{ \pm 1}$ of $P(t) / Q(t)$ is $n_{0}(d)$ (resp. $\left.n_{0}(d)-1\right)$ if $n$ is odd (resp. even). Hence, taking into account the reduction rules, the corollary follows.

4. Transversal surface maps. Throughout this section, $M$ will be a compact connected surface of genus $g$ and $f: M \rightarrow M$ a transversal map.

Recall that $H_{0}(M ; \mathbb{Q}) \approx \mathbb{Q}$ and that $H_{1}(M ; \mathbb{Q}) \approx \mathbb{Q}^{2 g}, H_{2}(M ; \mathbb{Q})$ $\approx \mathbb{Q}$ if $M$ is orientable, and $H_{1}(M ; \mathbb{Q}) \approx \mathbb{Q}^{g-1}, H_{1}(M ; \mathbb{Q}) \approx\{0\}$ if $M$ is non-orientable.

PRoposition 4.1. If $h(f)=0$ then all the eigenvalues of $f_{* 0}, f_{* 1}$ and $f_{* 2}$ are either 0 or roots of unity.

Proof. Since $f_{* 0}=\mathrm{id}, 1$ is the only eigenvalue of $f_{* 0}$.

Let us consider now $f_{* 2}$. If $M$ is non-orientable, then 0 is the only eigenvalue of $f_{* 2}$. If $M$ is orientable, $f_{* 2}(1)$ is the degree $D$ of $f$. From [MP] we know that if $|D|>1$ then $h(f) \geq \log |D|$. Hence, if $h(f)=0,|D| \leq 1$ and so the only possible eigenvalues for $f_{* 2}$ are $-1,0$ and 1 .

Finally, consider $f_{* 1}$. By Theorem 2 of [Mn], if $h(f)=0$ then all the eigenvalues $\lambda$ of $f_{* 1}$ satisfy $|\lambda| \leq 1$. We claim that every nonzero eigenvalue $\lambda$ of $f_{* 1}$ has modulus 1 . Let $\lambda_{1}, \ldots, \lambda_{k}$ be the nonzero eigenvalues of $f_{* 1}$. Then

$$
\operatorname{det}\left(I-t f_{* 1}\right)=\operatorname{det}\left(-t\left(f_{* 1}-t^{-1} I\right)\right)=(-1)^{k} \prod_{i=1}^{k}\left(\lambda_{i} t-1\right) .
$$

Moreover (4.1) must be a polynomial with integer coefficients, because $f_{* 1}$ is an integral matrix. Hence, in particular, $\prod_{i=1}^{k} \lambda_{i}$ must 
belong to $\mathbb{Z}$. Therefore, $\prod_{i=1}^{k}\left|\lambda_{i}\right| \geq 1$, and the claim follows because $\left|\lambda_{i}\right| \leq 1$.

So (4.1) is a polynomial with integer coefficients, constant term 1 and all its roots have modulus 1 . By a standard result in algebra (see Lemma 1.6 of [W]) the proposition follows.

COROllary 4.2. If $h(f)=0$ or $\operatorname{Per}(f)$ is finite, then $Z_{f}(t)$ is of the form

$$
Z_{f}(t)=\frac{\prod_{i=1}^{N} c_{n_{i}}(t)}{(1-t) \theta(t)}
$$

where $\theta(t)$ is either $1,1-t$ or $1+t$ and $c_{n_{t}}(t)$ is the $n_{i}$-cyclotomic polynomial.

Proof. The case $h(f)=0$ follows directly from the definition of the Lefschetz zeta function, Proposition 4.1 and Proposition 2.1. If $\operatorname{Per}(f)$ is finite, the corollary follows from Theorem 6 of [Fr].

The following results improve slightly the statements of Corollaries 3.3 and 3.4 for transversal surface maps.

Corollary 4.3. If $h(f)=0$ or $\operatorname{Per}(f)$ is finite, then

$$
\operatorname{Card}(\operatorname{FSP}(f)) \leq \begin{cases}g+1 & \text { if } M \text { is orientable, } \\ {\left[\frac{g-1}{2}\right]+1} & \text { if } M \text { is non-orientable. }\end{cases}
$$

Proof. From Corollary 4.2, repeating the arguments of the proof of Corollary 3.3 and taking into account that the contribution of $Q(t)=$ $Q_{2}(t)$ to $\operatorname{FSP}(f)$ is at most period 1 , the result follows.

The following result is just a restatement of Corollary 3.4 .

Corollary 4.4. If $h(f)=0$ or $\operatorname{Per}(f)$ is finite, then

$\operatorname{FSP}(f) \cap\{n \in \mathbb{N}: n$ is odd $\}$

$$
\subset \begin{cases}S\left(n_{0}(d)\right) & \text { if } n_{0}(d) \text { is odd }, \\ S\left(n_{0}(d)-1\right) & \text { if } n_{0}(d) \text { is even. }\end{cases}
$$

$\operatorname{FSP}(f) \cap\{n \in \mathbb{N}: n$ is even $\}$

$$
\subset \begin{cases}S\left(2 n_{0}(d)\right) & \text { if } n_{0}(d) \text { is odd, } \\ S\left(2 n_{0}(d)-2\right) & \text { if } n_{0}(d) \text { is even, }\end{cases}
$$

where $d=2 g$ if $M$ is orientable and $d=g-1$ if $M$ is non-orientable.

5. Transversal $N$-torus-maps. In this section we shall derive consequences of the results obtained in Section 3 for transversal self-maps 
$f$ on $n$-dimensional manifolds such that for $k=2, \ldots, n$ all the eigenvalues of $f_{* k}$ can be obtained as products of the eigenvalues of $f_{* 1}$. We remark that these conditions hold for continuous maps of the $n$-torus $T^{n}$ and also for continuous maps of many EilenbergMac Lane spaces.

The following corollary is easily obtained from Theorem 3.2 and Corollaries 3.3 and 3.4 repeating the arguments of the proof of Proposition 4.1 .

Corollary 5.1. Let $f: M \rightarrow M$ be a transversal map on a compact manifold $M$ of dimension $m$ with $h(f)=0$. Suppose that for $k=$ $2, \ldots, m$ all the eigenvalues of $f_{* k}$ can be obtained as products of the eigenvalues of $f_{* 1}$.

(a) If $Z_{f}(t)$ has an irreducible factor of the form $\left(1 \pm t^{n}\right)^{ \pm 1}$ with $n$ odd, then $n \in \operatorname{Per}(f)$.

(b) If $Z_{f}(t)$ has an irreducible factor of the form $\left(1 \pm t^{n}\right)^{ \pm 1}$ with $n$ even, then $\{n / 2, n\} \cap \operatorname{Per}(f) \neq \varnothing$.

(c) $\operatorname{Card}(\operatorname{FSP}(f)) \leq \operatorname{order}\left(Z_{f}(t)\right)$.

(d)

$$
\begin{aligned}
& \operatorname{FSP}(f) \cap\{n \in \mathbb{N}: n \text { is odd }\} \\
& \subset \begin{cases}S\left(n_{0}(d)\right) & \text { if } n_{0}(d) \text { is odd } \\
S\left(n_{0}(d)-1\right) & \text { if } n_{0}(d) \text { is even }\end{cases}
\end{aligned}
$$

(e)

$$
\begin{aligned}
\operatorname{FSP}(f) & \cap\{n \in \mathbb{N}: n \text { is even }\} \\
\subset & \quad \begin{cases}S\left(2 n_{0}(d)\right) & \text { if } n_{0}(d) \text { is odd, } \\
S\left(2 n_{0}(d)-2\right) & \text { if } n_{0}(d) \text { is even. }\end{cases}
\end{aligned}
$$

Similarly, using Theorem 3.2, Corollaries 3.3 and 3.4 and Theorem 4.1 of [ABLSS] we have the following result.

COROllary 5.2. Let $f: T^{m} \rightarrow T^{m}$ be a transversal map and suppose that $\operatorname{Per}(f)$ is finite. Then, (a) to (e) of Corollary 5.1 hold.

\section{REFERENCES}

[ABLSS] Ll. Alsedà, S. Baldwin, J. Llibre, R. Swanson and W. Szlenk, Minimal sets of periods for torus maps via Nielsen numbers, preprint of the Centre de Recerca Matemàtica (1991).

[CLN] J. Casasayas, J. Llibre and A. Nunes, Periodic orbits for transversal maps, preprint of the Centre de Recerca Matemàtica (1991). 
[F1] J. Franks, Some smooth maps with infinitely many hyperbolic periodic points, Trans. Amer. Math. Soc., 226 (1977), 175-179.

[F2] J. M. Franks, Homology and Dynamical Systems, CBMS Regional Conf. Series, Vol. 49, Amer. Math. Soc., 1982.

[F3] J. Franks, Period doubling and the Lefschetz formula, Trans. Amer. Math. Soc., 287 (1985), 275-283.

[Fr] D. Fried, Periodic Points and Twisted Coefficients, in Lecture Notes in Math., vol. 1007, Springer-Verlag (1983), 175-179.

[L] S. Lang, Algebra, Addison-Wesley, 1971.

[Mn] A. Manning, Topological Entropy and the First Homology Group, Lecture Notes in Math., vol. 468, Springer-Verlag (1975), 185-199.

[MP] M. Misiurewicz and F. Przytycki, Topological entropy and degree of smooth mappings, Bulletin de l'Académie Polonaise des Sciences, Série des Sciences Math., Astr. et Phys., XXV (1977), 573-578.

[Mt] T. Matsuoka, The number of periodic points of smooth maps, Ergodic Theory \& Dynamical Systems, 9 (1989), 153-163.

[W] L. C. Washington, Introduction to Cyclotomic Fields, Springer-Verlag, 1982.

Received December 16, 1991.

Departament de Matemàtica Aplicada i Anàlisi

UNIVERSITAT DE BARCELONA

Gran Via 585, 08071 Barcelona, Spain

Departament de MATemàtiques

Universitat Autònoma de Barcelona

Bellaterra, 08193 Barcelona, Spain

AND

Centre de Recerca Matemàtica

UNIVERSITAT AUTÓNOMA DE BARCELONA

Bellaterra, 08193 Barcelona, SPAIN

On leave from Departamento de Física, Universidade de lisboa

Campo Grande, Ed C1, Piso 4, 1700 Lisboa, Portugal 


\section{PACIFIC JOURNAL OF MATHEMATICS}

Founded by

E. F. BECKENBACH (1906-1982) F. Wolf (1904-1989)

\section{EDITORS}

Sun-Yung A. Chang

(Managing Editor)

University of California

Los Angeles, CA 90024-1555

chang@math.ucla.edu

\section{F. Michael Christ}

University of California

Los Angeles, CA 90024-1555

christ@math.ucla.edu

Herbert Clemens

University of Utah

Salt Lake City, UT 84112

clemens@math.utah.edu

\author{
ThOMAs ENRIGHT \\ University of California, San Diego \\ La Jolla, CA 92093 \\ tenright@ucsd.edu \\ Nicholas ERcolaNI \\ University of Arizona \\ Tucson, AZ 85721 \\ ercolani@math.arizona.edu \\ R. FINN \\ Stanford University \\ Stanford, CA 94305 \\ finn@gauss.stanford.edu \\ VAUGHAN F. R. Jones \\ University of California \\ Berkeley, CA 94720 \\ vfr@math.berkeley.edu
}

\author{
STEVEn KerCKHOFF \\ Stanford University \\ Stanford, CA 94305 \\ spk@gauss.stanford.edu
}

Martin ScharlemanN

University of California

Santa Barbara, CA 93106

mgscharl@math.ucsb.edu

\author{
Harold Stark \\ University of California, San Diego \\ La Jolla, CA 92093 \\ V. S. VARADARAJAN \\ University of California \\ Los Angeles, CA 90024-1555 \\ vsv@math.ucla.edu
}

\section{SUPPORTING INSTITUTIONS}

UNIVERSITY OF ARIZONA

UNIVERSITY OF BRITISH COLUMBIA

CALIFORNIA INSTITUTE OF TECHNOLOGY

UNIVERSITY OF CALIFORNIA

UNIVERSITY OF MONTANA

UNIVERSITY OF NEVADA, RENO

NEW MEXICO STATE UNIVERSITY

OREGON STATE UNIVERSITY

\author{
UNIVERSITY OF OREGON \\ UNIVERSITY OF SOUTHERN CALIFORNIA \\ STANFORD UNIVERSITY \\ UNIVERSITY OF HAWAII \\ UNIVERSITY OF UTAH \\ WASHINGTON STATE UNIVERSITY \\ UNIVERSITY OF WASHINGTON
}

The Supporting Institutions listed above contribute to the cost of publication of this Journal, but they are not owners or publishers and have no responsibility for its content or policies.

Mathematical papers intended for publication in the Pacific Journal of Mathematics should be in typed form or offset-reproduced (not dittoed), double spaced with large margins. Please do not use built up fractions in the text of the manuscript. However, you may use them in the displayed equations. Underline Greek letters in red, German in green, and script in blue. The first paragraph must be capable of being used separately as a synopsis of the entire paper. In particular it should contain no bibliographic references. Please propose a heading for the odd numbered pages of less than 35 characters. Manuscripts, in triplicate, may be sent to any one of the editors. Please classify according to the 1991 Mathematics Subject Classification scheme which can be found in the December index volumes of Mathematical Reviews. Supply name and address of author to whom proofs should be sent. All other communications should be addressed to the managing editor, or Julie Honig, University of California, Los Angeles, California 90024-1555.

There are page-charges associated with articles appearing in the Pacific Journal of Mathematics. These charges are expected to be paid by the author's University, Government Agency or Company. If the author or authors do not have access to such Institutional support these charges are waived. Single authors will receive 75 free reprints; joint authors will receive a total of 100 free reprints. Additional copies may be obtained at cost in multiples of 50 .

The Pacific Journal of Mathematics (ISSN 0030-8730) is published monthly except for July and August. Regular subscription rate: $\$ 215.00$ a year (10 issues). Special rate: $\$ 108.00$ a year to individual members of supporting institutions.

Subscriptions, orders for numbers issued in the last three calendar years, and changes of address should be sent to Pacific Journal of Mathematics, P.O. Box 4163, Berkeley, CA 94704-0163, U.S.A. Old back numbers obtainable from Kraus Periodicals Co., Route 100, Millwood, NY 10546.

The Pacific Journal of Mathematics at University of California, c/o Department of Mathematics, 981 Evans Hall, Berkeley, CA 94720 (ISSN 0030-8730) is published monthly except for July and August. Second-class postage paid at Berkeley, CA 94704, and additional mailing offices. POSTMASTER: send address changes to Pacific Journal of Mathematics, P.O. Box 4163, Berkeley, CA 94704-0163.

PUBLISHED BY PACIFIC JOURNAL OF MATHEMATICS at University of California, Berkeley, CA 94720, A NON-PROFIT CORPORATION

This publication was typeset using $\mathcal{A} \mathcal{M} \mathcal{S}-\mathrm{T}_{\mathrm{E}} \mathrm{X}$,

the American Mathematical Society's $T_{E} X$ macro system.

Copyright (c) 1994 by Pacific Journal of Mathematics 


\section{PACIFIC JOURNAL OF MATHEMATICS}

Volume $165 \quad$ No. $1 \quad$ September 1994

The effect of dimension on certain geometric problems of irregularities of 1 distribution

RALPH ALEXANDER

The structure of $\mathrm{sl}(2,1)$-supersymmetry: irreducible representations and primitive ideals

Didier ARnAL, HÉdi BENAMOR and GEORges PinCZON

Periods and Lefschetz zeta functions

Josefina CASASAYas, JaUme Llibre and ANA Nunes

On closed hypersurfaces of constant scalar curvatures and mean curvatures in 67 $S^{n+1}$

\section{SHAOPING CHANG}

$R$-groups and elliptic representations for $\mathrm{SL}_{n}$

DAVID GOLDBERG

The boundary distortion of a quasiconformal mapping

JuHA HeINONEN and PEKKA KosKela

Strongly approximately transitive group actions, the Choquet-Deny theorem, 115 and polynomial growth

\section{WOJCIECH JAWORSKI}

$q$-canonical commutation relations and stability of the Cuntz algebra

PALle E. T. Jorgensen, L. M. Schmitt and Reinhard Frank

WERNER

Complete open manifolds of non-negative radial curvature

YOSHIROH MACHIGASHIRA

Perturbations of certain reflexive algebras

DAVID RYDER PITTS

Interpolation submanifolds of the unitary group 